\title{
Quality Evaluation of Voice Transmission Using BPL Communication System in MV Mine Cable Network
}

\author{
Grzegorz Debita ${ }^{1, *}$, Przemyslaw Falkowski-Gilski² ${ }^{2}$ Marcin Habrych ${ }^{3}$, Bogdan Miedzinski ${ }^{4}$, \\ Janusz Wandzio ${ }^{5}$, Przemyslaw Jedlikowski ${ }^{6}$ \\ ${ }^{1}$ General Tadeusz Kosciuszko Military University of Land Forces, \\ Czajkowskiego 109, 51-147 Wroclaw, Poland \\ ${ }^{2}$ Faculty of Electronics, Telecommunications and Informatics, Gdansk University of Technology, \\ Narutowicza 11/12, 80-233 Gdansk, Poland \\ ${ }^{3}$ Faculty of Electrical Engineering, Wroclaw University of Science and Technology, \\ Wybrzeże Wyspiańskiego 27, 50-370 Wroclaw, Poland \\ ${ }^{4}$ Institute of Innovative Technologies EMAG, \\ 40-189 Katowice ul Leopolda 31, Poland \\ ${ }^{5}$ KGHM Polska Miedz S.A., \\ Sklodowskiej-Curie 48, 59-301 Lubin, Poland \\ ${ }^{6}$ Faculty of Electronics, Wroclaw University of Science and Technology, \\ Wybrzeże Wyspiańskiego 27, 50-370 Wroclaw, Poland \\ grzegorz.debita@awl.edu.pl
}

\begin{abstract}
This article presents results of a quality evaluation study, considering voice transmission in a $6 \mathrm{kV}$ medium voltage cable network using the BPL (Broadband over Power Line) communication system. The tests are carried out under real mining conditions for the selected power cable without voltage, earthed at both sides. Such a method of monitoring work conditions is of great importance, especially during a disaster. Power cables are potentially resistant to mechanical damage and, thus, ensure continuity of transmission, also, in cases of electricity stoppage and partial damage (interruption) of the phase conductors. The voice transmission quality is tested for a relatively low bitrate of 8 kbps-48 kbps using both induction and mixed inductioncapacitive coupling with the power cable. Such a solution should provide both reliable and high-quality services, considering clear and understandable voice messages to people. The quality evaluation is carried out on a group of 15 people aged between $25-35$ years old. The tested signal samples consist of voice messages in British English, German, and Polish. On the basis of the investigated results, respective conclusions are formulated.
\end{abstract}

Index Terms-BPL communication system; MV cable network; Quality evaluation; Signal processing; Voice transmission.

\section{INTRODUCTION}

Reliable and efficient transmission of different types of content is a crucial requirement for any underground mine system. In order to provide secure operating conditions for people, various types of transmissions, both wired and wireless, are used and duplicated for safety purposes.

Manuscript received 6 March, 2019; accepted 10 July, 2019.
Recently, the transmission of so-called power line communication (PLC) from low (up to $150 \mathrm{kHz}$ ) to high (between $3 \mathrm{MHz}-32 \mathrm{MHz}$ ) frequency (BPL - Broadband over Power Line) is gaining popularity [1]-[4]. Its main advantage is the possibility to utilize the existing infrastructure of electric networks, both low and high voltage conductors, as well as mine cables without the need to incur additional costs related to operator's fees.

In addition, the probability of a total mechanical damage of the mining cable, especially the medium voltage one, under various sidewalk and pit collapse incidents seems to be relatively low. The BPL transmission is then performed through the armor and/or shielding of the cable with the interrupted phase conductors. This applies especially to the inductive coupling of modems with the cable when using batteries. Whenever an emergency situation occurs, particularly in the underground environment, it is crucial to ensure and maintain the continuity of the voice transmission.

In this paper, we focus on transmitting voice messages of an appropriate quality using the BPL communication system of a medium voltage cable $(6 \mathrm{kV})$ under real operating conditions in an underground mine environment, what is a scientific novelty of the paper. The unidirectional transmission tests are performed in a scenario when the $6 \mathrm{kV}$ cable operates under emergency (lack of voltage, short circuit, earthed cable input and output). A suitable set of a digital transmitter and receiver connected to BPL modems are developed for this purpose. Note, that nowadays, due to the bandwidth restrictions, the main goal of any digital communication system is to deliver high-quality content with the lowest possible bitrate. This bitrate depends on a set of 
factors, including the type of medium, delay related with distance, error rate, etc.

Effective BPL transmission also requires the selection of the appropriate mode (bandwidth) depending on the cable parameters (characteristic impedance, attenuation, etc.) and the optimal operation of the coupling used [1]-[4]. For this reason, tests are carried out for in the range of frequency of $2 \mathrm{MHz}-7 \mathrm{MHz}$ and at the different bitrates for voice transmission up to $48 \mathrm{kbps}$. The evaluation of voice quality is carried out on a selected group of people. Then, obtained data is subjected to the statistical treatment. Based on these results, practical conclusions are formulated.

\section{UNDERGROUND ENVIRONMENTS}

The cable feeder with the installed BPL communication system consisted of several different types of cables connected to each other at the respective substation busbars. It was located both in the shaft and the underground pavement at a depth of approximately $1100 \mathrm{~m}$ as shown in Fig. 1.

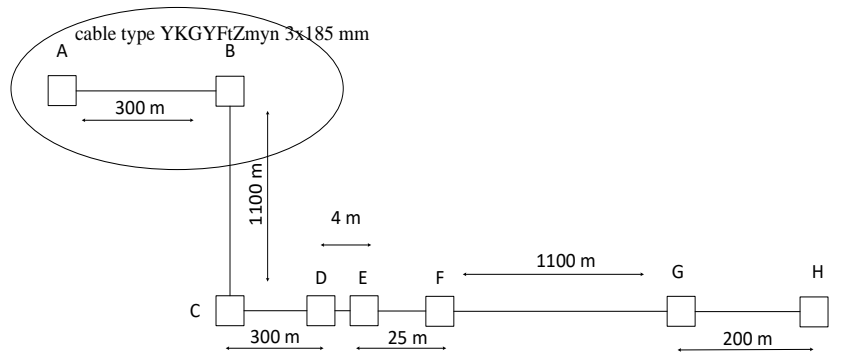

Fig. 1. Tested $6 \mathrm{kV}$ cable line with marked section of the selected test cable located in the mine shaft headroom.

Each section of the line was, therefore, exposed to different environmental conditions (temperature and humidity). A section of the YKGYFtZmyn 3 x $185 \mathrm{~mm}$ cable with a medium voltage line of $6 \mathrm{kV}$, about $300 \mathrm{~m}$ long, located in the mine shaft head was selected for the tests as indicated in Fig.1. The ambient temperature during the tests was equal to $11.4^{\circ} \mathrm{C}$, whereas humidity did not exceed $60 \%$.

\section{ABOUt THE TEST}

In the proposed scenario, tests were carried out for the unilateral voice transmission from point A to point B of the cable line located in the mine shaft headroom. Test was performed under the operating power line $(6 \mathrm{kV}$, current around 25 Arms, $\mathrm{THD}_{\mathrm{u}}$ up to $3 \%, \mathrm{THD}_{\mathrm{i}}$ around $10 \%$ ) and without voltage for its earthing at both sides. Both induction coupling of the BPL modems with a cable on both sides (Fig. 2) and capacitive-induction ones (Fig. 3) were used. The method of installing capacitive couplers (at point A), and induction couplers (at points $\mathrm{A}$ and $\mathrm{B}$ ) is shown in Fig. 4-Fig. 6, respectively.

The PC located at point A with the UBUNTU operating system worked as the server with the IC CAST software installed. Whereas, at point $\mathrm{B}$, the Raspberry Pi microcomputer was used (due to spatial limitations in the mine's sidewalk) with the RASBIAN operating system installed. It performed as the client, along with the VLC software. Voice samples received from the server were recorded at the Pi client side.

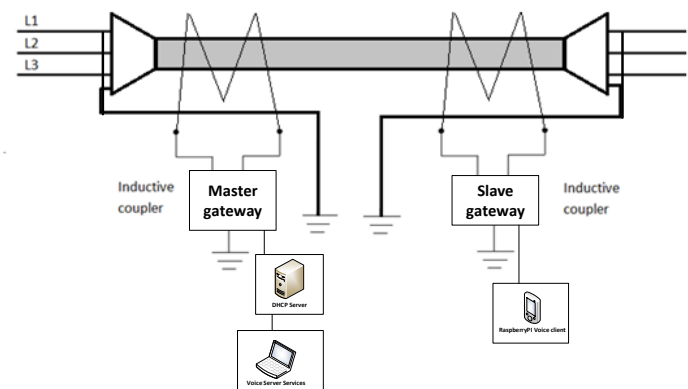

Fig. 2. Tested connection for induction-induction coupling (Test 2).

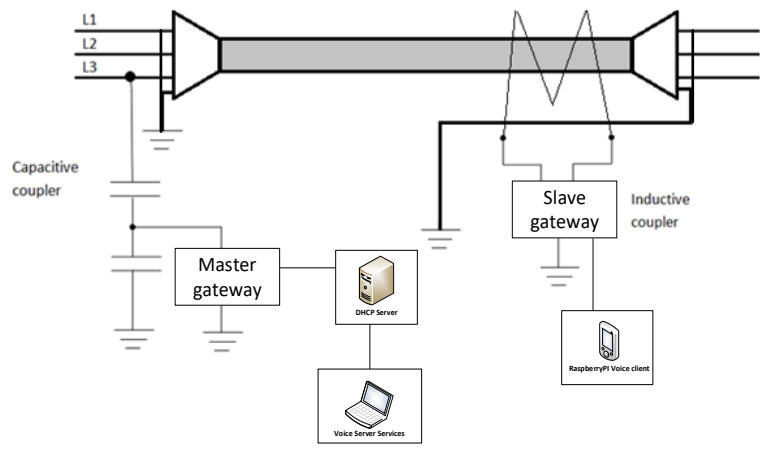

Fig. 3. Tested connection for capacitive-induction coupling (Test 1)

The developed transmitter and digital receiver were connected to BPL modems, respectively (Fig. 2 and Fig. 3). The BPL transmission system operated in the frequency range of $2 \mathrm{MHz}-7 \mathrm{MHz}$, whereas the bitrate of voice services ranged from $8 \mathrm{kbps}-48 \mathrm{kbps}$. A single session, considering sending, receiving, and recording voice messages for each of the selected 5 different bitrates $(8 \mathrm{kbps}$, $16 \mathrm{kbps}, 24 \mathrm{kbps}, 32 \mathrm{kbps}$, and $48 \mathrm{kbps}$ ) took about 10 minutes, respectively.

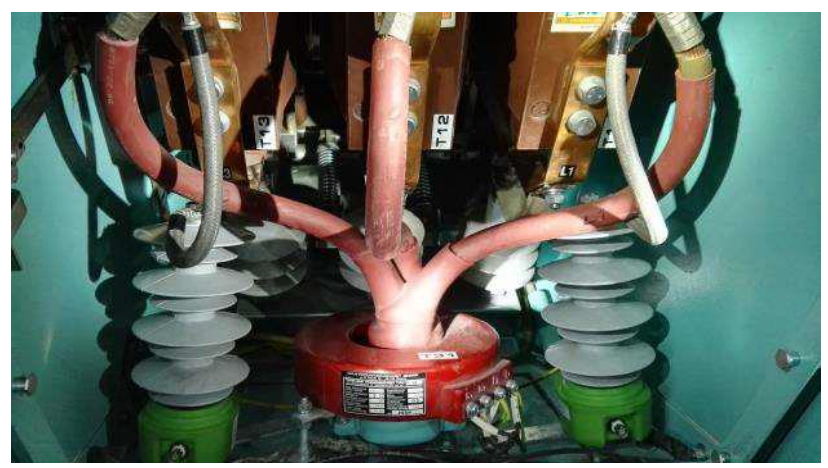

Fig. 4. Capacitive couplers installed at point A.

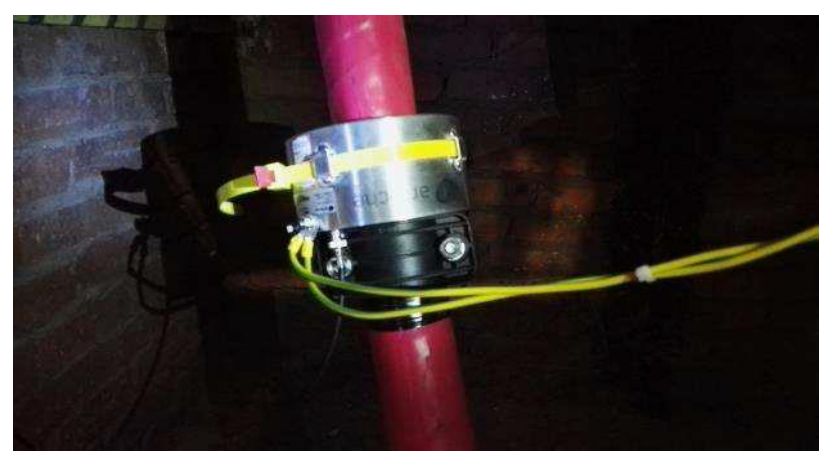

Fig. 5. Inductive couplers installed at point A. 


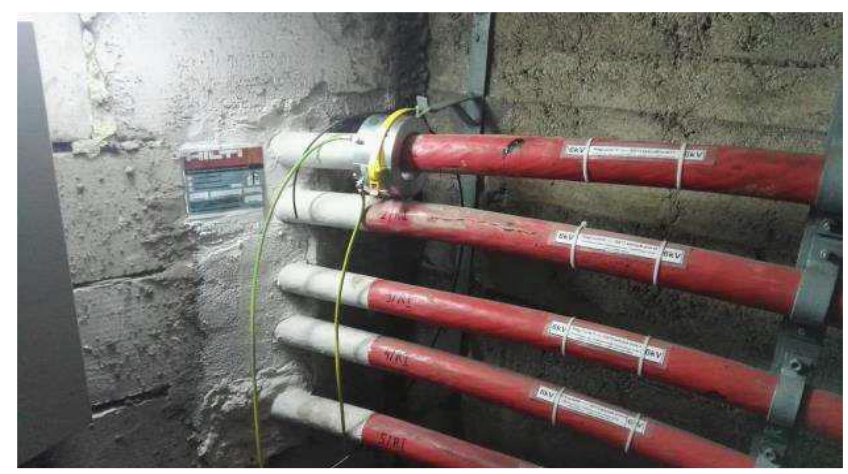

Fig. 6. Inductive couplers installed at point B.

\section{QUALITY EVALUATION}

The human perception differs from person to person. In this test, a group of 15 people aged between 25-35 years old is selected. This group meets the average age of workers present in the company. Of course, any subjective judgement is affected not only by the sound quality itself, but also age and hearing defects of an individual. In this case, none of them has hearing disorders.

In the proposed scenario, the system should provide clear and understandable voice messages to people. In order to design such a system, one needs to know how many bits are sufficient to deliver such a message. Moreover, the bandwidth fluctuations, the packet loss or the delay can cause the degradation in quality understood as network QoS (Quality of Service) or user QoE (Quality of Experience).

\section{A. Signal Samples}

For the purpose of this test, a set of signal samples were selected. These files were sourced from ITU (International Telecommunication Union) [5]. The description of the selected speech samples in British English, German, and Polish from Annex B (Speech and Noise Signals Clause B) is shown in Table I.

From the set of available languages, both male and female samples were utilized. The sourced audio material, of $7 \mathrm{~s}-8 \mathrm{~s}$ duration each, was WAV 16-bit PCM (Pulse Code Modulation) files with sampling frequency set to $32 \mathrm{kHz}$. Each file was then processed using the OGG coding algorithm, transmitted though the wired medium, and recorded on the receiving side.

TABLE I. SPEECH SIGNALS USED DURING TEST

\begin{tabular}{|c|c|}
\hline File name & \multirow{3}{*}{ Language } \\
\hline ENfemale1 & \multirow{2}{*}{ British English } \\
\hline ENfemale2 & \\
\hline ENmale1 & \\
\hline ENmale2 & German \\
\hline GEfemale1 & \\
\hline GEfemale2 & \\
\hline GEmale1 & \\
\hline GEmale2 & Polish \\
\hline PLfemale1 & \\
\hline PLfemale2 & \\
\hline PLmale1 & \\
\hline PLmale2 &
\end{tabular}

The speech signal samples were processed in 5 different bitrates, namely: $8 \mathrm{kbps}, 16 \mathrm{kbps}, 24 \mathrm{kbps}, 32 \mathrm{kbps}$, and $48 \mathrm{kbps}$. Previous studies concerning delivering speech audio signals showed that a bitrate of $48 \mathrm{kbps}-64 \mathrm{kbps}$ enables to deliver high-quality commercial services [6]-[8]. Additional information on speech signal processing may be found in [9].

\section{B. Subjective Quality Assessment}

Tests were carried out in turns, one participant after another, according to [10]. A single session took approximately $20 \mathrm{~min}$. All 15 listeners took a training phase before starting the essential study in order to become familiar with the test scenario and acquaint with the listening equipment. The subjective quality was assessed using the Beyerdynamic Custom One Pro headphones. Further information on testing audio signals may be found in [11], whereas the principles considering speech coding, especially at low bitrates, are available in [12]-[14].

However, after a few tests, the number of evaluated signal samples was limited to 3 bitrates, i.e., $8 \mathrm{kbps}, 16 \mathrm{kbps}$, and $24 \mathrm{kbps}$. Quite surprisingly, the subjective judgements for bitrates higher than $24 \mathrm{kbps}$ did not differ much. That is why the bitrates of $32 \mathrm{kbps}$ and $48 \mathrm{kbps}$ were omitted from the assessment. Due to this fact, a single session was limited to approximately 12 minutes.

The results of the subjective quality evaluation concerning the overall quality of the voice messages transmitted over the BPL cable network in British English, German, and Polish are shown in Figs. 7-9. In this case, Test 1 refers to the communication using capacitive-induction coupling, whereas Test 2 refers to the case of the inductive-inductive coupling.

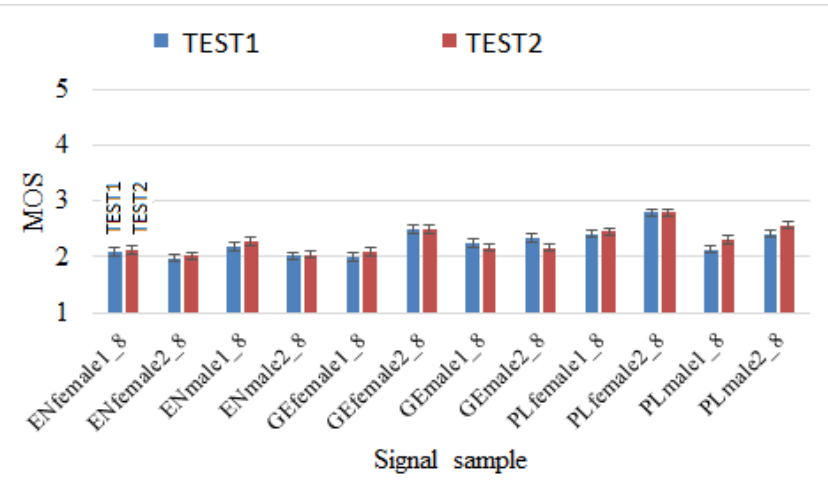

Fig. 7. Quality evaluation for speech signal samples transmitted at $8 \mathrm{kbps}$.

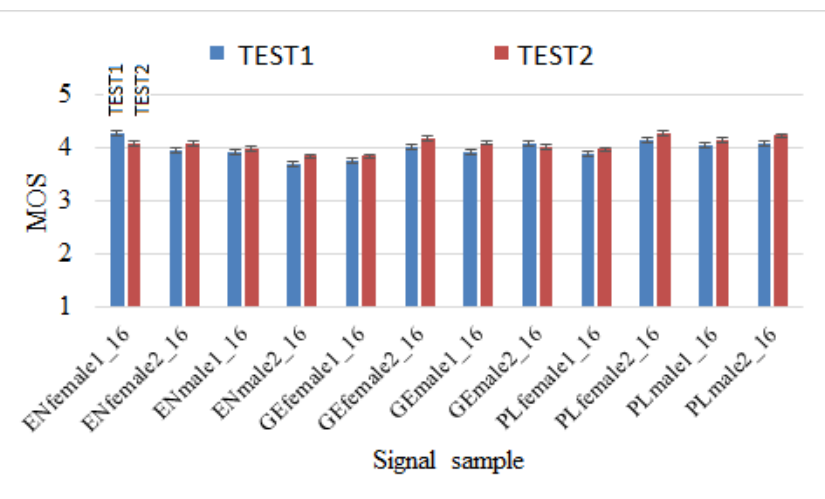

Fig.8. Quality evaluation for speech signal samples transmitted at $16 \mathrm{kbps}$.

The obtained subjective results were treated with the ANOVA (Analysis of Variance) statistical analysis as shown in Table II. The confidence interval $\alpha$ was set to $95 \%$, whereas $F_{\text {crit }}$ was equal to 1.75 . 


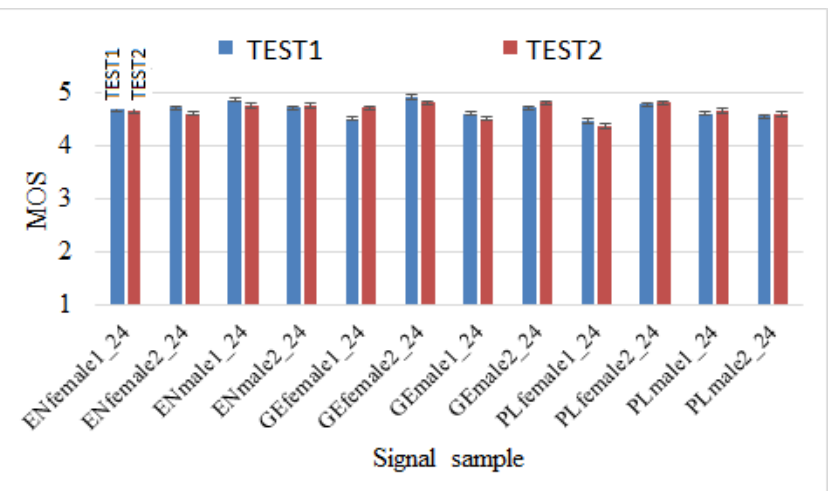

Fig. 9. Quality evaluation for speech signal samples transmitted at $24 \mathrm{kbps}$.

TABLE II. ANOVA TEST RESULTS.

\begin{tabular}{|c|c|c|}
\hline Data set & P & F \\
\hline Capacitive coupling 8 kbps & 0.08 & 2.26 \\
\hline Capacitive coupling 16 kbps & 0.30 & 1.18 \\
\hline Capacitive coupling 24 kbps & 0.67 & 0.81 \\
\hline Inductive coupling 8 kbps & 0.12 & 1.48 \\
\hline Inductive coupling 16 kbps & 0.79 & 0.69 \\
\hline Inductive coupling 24 kbps & 0.46 & 1.00 \\
\hline
\end{tabular}

According to the obtained results, in each case the $P$ value was not less than $\alpha$. Additionally, the $F$ value did not exceed $F_{\text {crit. }}$

\section{CONCLUSIONS}

Studies show, that in case of broadcasting and streaming services, speech signals are perceived as of high quality for a bitrate of $48 \mathrm{kbps}-64 \mathrm{kbps}$, whereas, for music signals, the required bitrate ranges from $96 \mathrm{kbps}$ to $128 \mathrm{kbps}$. However, this bitrate is necessary for regular commercial audio services. When transmitting voice messages in an underground communication system, the bandwidth requirements can be lowered.

In case of the lowest bitrate of $8 \mathrm{kbps}$, due to high distortions, sentences spoken by both male and female lectors prove to be unacceptable. Furthermore, as indicated by the listeners, the female voice is found to be more understandable. For $16 \mathrm{kbps}$, an increase in subjective quality is clearly noticeable. However, not all samples are perceived as of good quality. According to the obtained results, a clear and understandable voice message can be provided for a bitrate equal to $24 \mathrm{kbps}$. High overall quality resembling a MOS score of 4.0 and above is observed regardless of the spoken language.

The spoken language of the presented voice messages, whether it is British English, German, or Polish, does not have a significant impact on the results obtained. In case of each participant, Polish language is indicated as the mother tongue, whereas English is the second language of choice, in which they most often communicate abroad. German is the third language of choice. Additionally, as pointed out by the listeners, sentences spoken by a male lector seem more appealing.

This is independent of the type of BPL modem coupling used with the mining cable. In general, when it comes to maintaining a connection between the supervisor and the crew members, it can be concluded, based on the subjective judgements, that the male voice is preferred more. Nevertheless, the topic of the designing high-quality and reliable services considering different types of wired media still remains a challenge.

\section{REFERENCES}

[1] P. Mlynek, J. Misurec, P. Toman, P. Silhavy, R. Fujdiak, J. Slacik, Z. Hasirci, K. Samouylov, "Performance Testing and Methodology for Evaluation of Power Line Communication", Elektronika ir Elektrotechnika, vol. 24, no. 3, pp. 88-95, 2018. DOI: 10.5755/j01.eie.24.3.20983.

[2] P. Mlynek, J. Misurec, and M. Koutny, "Modeling and evaluation of power line for Smart grid communication", Prz. Elektrotech., vol. 87, no 8, pp. 228-232, 2011.

[3] G. Debita, M. Habrych, A. Tomczyk, B. Miedzinski, and J. Wandzio, "Implementing BPL transmission in MV cable network effectively", Elektronika ir Elektrotechnika, vol. 25, no. 1, pp. 59-65, 2019. DOI: 10.5755/j01.eie.25.1.22737.

[4] H. Meng, S. Chen, Y. L. Guan, C. L. Law, P. L. So, E. Gunawan, and T. T. Lie, "Modeling of transfer characteristics for the broadband power line communication channel", IEEE Tran. Power Del., vol. 19, pp. 1057-1064, 2004. DOI: 10.1109/TPWRD.2004.824430.

[5] Test Signals for Telecommunication Systems, ITU-T Recommendation P.501, 2017.

[6] P. Gilski and J. Stefanski, "Subjective and objective comparative study of DAB+ broadcast system", Arch. Acoust., vol. 42, no. 1, pp. 3-11, 2017. DOI: 10.1515/aoa-2017-0001.

[7] P. Gilski, "DAB vs $\mathrm{DAB}+$ radio broadcasting: a subjective comparative study", Arch. Acoust., vol. 42, no. 4, pp. 157-165, 2017. DOI: 10.1515/aoa-2017-0074.

[8] K. Ulovec and M. Smutny, "Perceived audio quality analysis in digital audio broadcasting plus system based on PEAQ", Radioengineering, vol. 27 , no. 1, pp. 342-352, 2018. DOI: 10.13164/re.2018.0342.

[9] S. Brachmanski, "Quality evaluation of speech AAC and HE-AAC coding", in Proc. Joint Conference - Acoustics 2018, Ustka, 2018, pp. 1-4. DOI: 10.1109/ACOUSTICS.2018.8502335

[10] General methods for the subjective assessment of sound quality, ITU-R Recommendation BS.1284, 2003.

[11] S. Brachmanski, Selected issues in assessing the quality of speech transmission [in Polish: Wybrane zagadnienia oceny jakości transmisji sygnału mowy]. Wroclaw, Poland, Oficyna Wydawnicza Politechniki Wroclawskiej, 2015.

[12] J. G. Proakis, Wiley Encyclopedia of Telecommunications. Hoboken, NJ, John Wiley \& Sons, 2003. DOI: 10.1002/0471219282.

[13] M. Yang, "Low bit rate speech coding", IEEE Potentials, vol. 23, no. 4, pp. 32-36, 2004. DOI: 10.1109/MP.2004.1343228.

[14] J. Benesty, M. M. Sondhi, and Y. Huang, Springer Handbook of Speech Processing. Berlin, Germany: Springer, 2008. DOI: 10.1007/978-3-540-49127-9. 